\title{
Renal replacement therapy in acute kidney injury from a Chinese cross-sectional study: patient, clinical, socioeconomic and health service predictors of treatment
}

Fang Wang ${ }^{1+}$, Daqing Hong ${ }^{1+} \mathbb{D}$, Yafang Wang ${ }^{2,3 \dagger}$, Yunlin Feng ${ }^{1}$, Li Wang ${ }^{1 *}$, Li Yang ${ }^{2,3^{*}}$ and ISN AKF 0 by 25 China Consortium

\begin{abstract}
Background: Renal replacement therapy (RRT) is important to support critically ill patients with acute kidney injury (AKI). This study, a part of a nation-wide survey for AKI conducted by the ISN AKF 0 by 25 China Consortium, aims to study the current RRT practical situation and problems in China.

Methods: The current study is a part of a nation-wide survey for AKI conducted by ISN AKF 0 by 25 China Consortium. The survey included 44 sites all over the country, including 22 academic hospitals in big cities and 22 local hospitals in smaller cities or rural areas. Of the 44 sites, all have access to PD and IHD, 93.5\% are capable to perform CRRT. Of total 7604 AKI cases, 896 cases (11.8\%) had indications for RRT and were included in the current abstract.

Results: of the 896 patients that had indications for RRT, only 59.3\% received RRT. Patients who were older, male, from lower income areas, in local hospitals, or with severe comorbidities, were less likely to receive RRT. RRT treatment was associated with lower mortality $(\mathrm{OR}=0.58,95 \% \mathrm{Cl} 0.38-0.89)$. The RRT modalities were continuous renal replacement therapy (CRRT) in 53.9\%, intermittent hemodialysis (IHD) in 38.0\%, CRRT complemented by IHD in 6.2\%, CRRT complemented by peritoneal dialysis (PD) in $0.8 \%$ and PD in $1.1 \%$. Of the subgroup of patients receiving RRT who did not have an indication for modality of CRRT, $36.8 \%$ in fact received CRRT, and their medical cost and mortality rate was higher $(7944[4248,16,055]$ vs. 5100[2948, 9396] US dollars, $p<0.001$ and 10.6\% vs. 4. $4 \%, p=0.047$, respectively) compared with those treated with other RRT modalities).

Conclusions: Extrapolated to the whole of China our results indicate that an estimated 139,000 patients with an indication of RRT are under treated without RRT over a year. Non-clinical factors influence RRT prescription for severe AKI patients. CRRT may be over-utilized in the treatment of severe AKI and the use of PD is extremely rare. These findings have implications for the effective application of medical resources in the treatment of severe AKI.
\end{abstract}

Keywords: Acute kidney injury, Hemodialysis, Peritoneal dialysis, Renal replacement therapy, Developing countries, China

\footnotetext{
* Correspondence: scwangli62@163.com; li.yang@bjmu.edu.cn

${ }^{\dagger}$ Equal contributors

'Division of Nephrology, Sichuan Academy of Medical Sciences \& Sichuan

Provincial People's Hospital, Chengdu 610072, China

${ }^{2}$ Renal Division, Department of Medicine, Peking University First Hospital,

Beijing 100034, People's Republic of China

Full list of author information is available at the end of the article
} 


\section{Background}

Acute kidney injury (AKI), a "Silent killer" [1], is getting more and more attention because of its increasing incidence and adverse impact on patients' outcome and health cost burden [2-7]. However, AKI are still underrecognized and/or under-treated, especially in the developing countries, due to low awareness and low medical resources [8].

Renal replacement therapy (RRT), as a supportive management, remains the main treatment strategy for severe AKI patients. Although debates on the optimal timing to initiate RRT and the optimal choice of RRT modalities still continue, knowledge must be known about the current situation lagging behind the strategy available nowadays, and efforts need to be made to improve this situation which is as important as optimizing the management which is relied on future research.

In order to improve the diagnosis and treatment of AKI globally, International Society of Nephrology (ISN) carried out a global target of 0 by 25 -zero death of patients with untreated acute kidney failure by 2025. As part of this project, we carried out the largest nationwide survey for AKI in China. In order to reveal the current status and further improve the situation of RRT practice for the AKI patients in China, we performed a sub-analysis on the data collected from the survey, including 2,223,230 hospitalized adult patients from 22 provinces, municipalities or autonomous regions in Mainland China [9].

\section{Methods}

\section{Participants}

Patients were derived from a cross-sectional survey from 22 Chinese provinces, municipalities or autonomous regions, which covered $82 \%$ of the country's population and four geographic regions of China (North, Northwest, Southwest, and Southeast) [9]. 2,223,230 adult patients ( $>=18$ years) were admitted in 44 study hospitals during 2013, among which 26,086 cases were reviewed with medical records. AKI was diagnosed in 7064 cases, of which 896 patients with renal replacement therapy indications were finally enrolled in this sub-analysis (Study profile, Fig. 1). All the study hospitals that were enrolled in the nation-wide survey were general hospitals with nephrology specialty and facilities for hemodialysis (HD), and peritoneal dialysis (PD). Continuous renal replacement therapy (CRRT) was available in $93.5 \%$ of the hospitals. The study protocol and waiver of patient informed consent was approved by the ethic committees of Peking University First Hospital and the enrolled study hospitals. Required data for this study was obtained in a de-identified and anonymized form.

\section{Diagnosis criteria}

Three steps were carried out to accomplish this national survey. Firstly, serum creatinine $(\mathrm{SCr})$ was reported by the Laboratory Information System and changes in serum creatinine $(\triangle \mathrm{SCr})$ were evaluated to screen AKI. Secondly, hospital records of the suspected patients were reviewed by trained nephrologists/renal fellows to confirm the diagnosis of AKI. Thirdly, relevant records (such as demographic information, comorbidities, clinical departments, in-hospital costs and outcomes, etc.) were completed for the patients confirmed with AKI.

AKI was diagnosed according to either 2012 KDIGO AKI definition [10] (criteria 1) or an expanded criterion as defined by authors: an increase or decrease in SCr by $50 \%$ during hospital stay (criteria 2). Patients with baseline CKD stage 5, previous nephrectomy, kidney transplantation, peak $\mathrm{SCr}<0.6 \mathrm{mg} / \mathrm{dl}$ or changes of $\mathrm{SCr}$ not attributed to AKI (such as SCr decrease after amputation, etc.) were excluded. Severe comorbidity was defined as having either of the following clinical situations: multiple organ dysfunction syndrome (MODS), disseminated intravascular coagulation, sepsis, advanced stage of malignancies, acute respiratory distress syndrome, shock or mechanical ventilation.

The indications for RRT included1) volume overload unresponsive to diuretic therapy; or 2) severe hyperkalemia ( $\geq 6 \mathrm{mmol} / \mathrm{L})$ or metabolic acidosis $(\mathrm{pH} \leq 7.3)$; or 3 ) BUN $\geq 60 \mathrm{mmol} / \mathrm{L}$ or 4 ) overt uremic manifestations such as pericarditis and encephalopathy. In additions to the above traditional indications for CRRT, if patients had at least one of the following commobidities: rhabdomyolysis, sepsis, MODS, respiratory failure requiring mechanical ventilation, shock, CRRT were also indicated as RRT could potentially benefits these patients.

Renal recovery at discharge was classified as: 1) full recovery, defined as SCr fell below threshold or to the baseline; 2) partial recovery, defined as $\mathrm{SCr}$ decreased by $\geqq 25 \%$ from peak level but remained above the threshold or baseline; 3) failure to recover, defined as dialysis dependent or SCr decreased by $<25 \%$ from peak level. In-hospital mortality was defined as recorded death in medical records. Details in the study design and working process was present in the previous publication [9].

\section{Statistical analysis}

Continuous data were presented as means with SDs or medians (IQR). Categorical variables were presented as proportions. Continuous data were compared between two groups using t-test or non-parametric Wilcoxon rank sum test for severely skewed data. Chi-square test was used to compare categorical variables between two groups. In this study, demographic characteristics, comorbidities, in-hospital covariates (such as admission department, renal consultancy, etc.), degree of disease, 


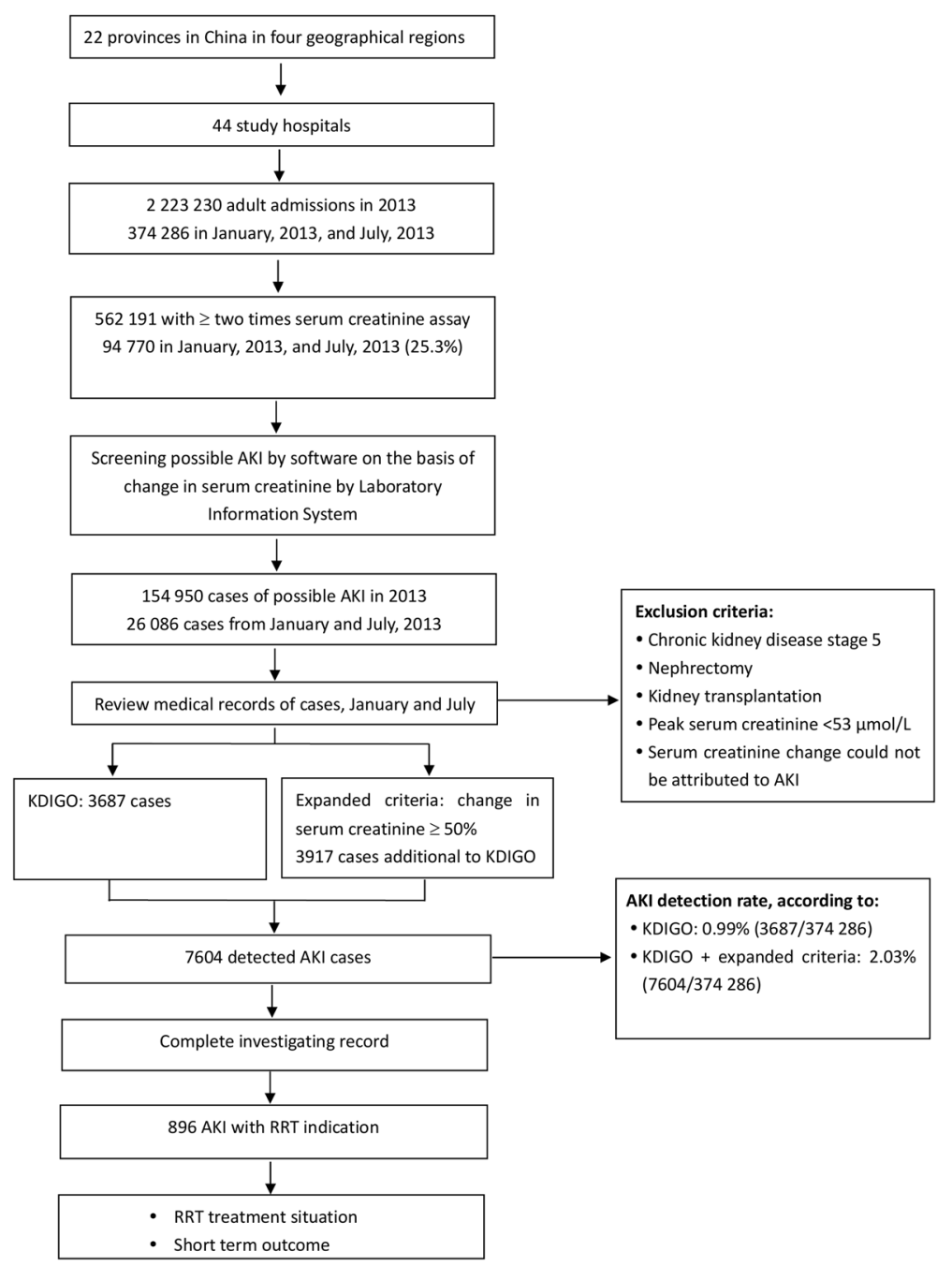

Fig. 1 Study profile. Abbreviations: AKl, acute kidney injury; RRT, renal replacement therapy. Notes: This figure was modified with permission from the original report (9)

renal recovery, in-hospital mortality and costs were compared between RRT treatment group and no-RRT treatment group. Multiple logistic regression was applied to study the factors associated with no-RRT treatment using variables including age, gender, gross domestic product (GDP) per head, academic hospital, renal referral, AKP peak stage, hospital-acquired AKI, non-oliguria and severe comorbidities. The relationship between RRT and in-hospital mortality was analyzed with multiple logistic regression adjusting for age, gender, GDP per head, academic hospital, renal referral, AKI peak stage, hospital-acquired AKI, cardiovascular disease (CVD), diabetes mellitus (DM), non-oliguria and severe comorbidities. RRT modalities were divided into CRRT, CRRT + IHD, CRRT + PD, IHD and PD, and Chi-square was utilized to compare the difference of RRT modalities among departments including ICU, renal and other departments. Age, gender, AKI peak stage, severe comorbidities, costs, renal referral, in-hospital and short-term mortality and cost were compared between CRRT and other-RRTtreated groups in patients without CRRT indications. A two tailed $P$ value $<0.05$ was regarded as indicative of statistical significance. All analyses were performed using SAS software (version 9.1, SAS Institute, Cary, NC, USA).

\section{Results}

Characteristics of participants with RRT indications (Table 1)

Of total 7604 AKI cases, 896 cases (11.8\%) had indications for RRT and were included in the current study. The mean age was $59.7 \pm 18.2$ years. $65.5 \%$ were male patients baseline chronic kidney disease (CKD) was in $34.2 \%$ of patients. $41.5 \%$ of patients had hypertension while 20.0\%haddiabetes mellitus Most of the patients were hospitalized in academic hospitals (81.7\%). The percentage of the patients who were admitted to intensive care unit (ICU), renal, surgical and other medical 
Table 1 Characteristics of participants with RRT indications

\begin{tabular}{|c|c|c|c|c|}
\hline & Total $(n=896)$ & $\operatorname{RRT}(n=531)$ & No-RRT $(n=365)$ & $P$ \\
\hline Age, years & $59.7 \pm 18.2$ & $57.0 \pm 18.2$ & $63.7 \pm 17.4$ & $<0.001$ \\
\hline Male sex & $587(65.5 \%)$ & $322(60.6 \%)$ & $265(72.6 \%)$ & $<0.001$ \\
\hline GDP per head ${ }^{a}$ & & & & $<0.001$ \\
\hline Tertile 1 & $294(32.8 \%)$ & $139(26.2 \%)$ & $155(42.5 \%)$ & \\
\hline Tertile 2 & 319 (35.6\%) & $192(36.2 \%)$ & $127(34.8 \%)$ & \\
\hline Tertile 3 & $283(31.6 \%)$ & $200(37.7 \%)$ & $83(22.7 \%)$ & \\
\hline HA- AKI & $360(40.2 \%)$ & $184(34.7 \%)$ & $176(48.2 \%)$ & $<0.001$ \\
\hline CKD & $306(34.2 \%)$ & $185(34.8 \%)$ & $121(33.2 \%)$ & 0.600 \\
\hline $\mathrm{HT}$ & $372(41.5 \%)$ & $214(40.3 \%)$ & $158(43.3 \%)$ & 0.373 \\
\hline $\mathrm{DM}$ & $179(20.0 \%)$ & $106(20.0 \%)$ & $73(20.0 \%)$ & 0.989 \\
\hline CVD & $267(29.8 \%)$ & $138(26.0 \%)$ & $129(35.3 \%)$ & 0.003 \\
\hline Malignancies & $143(16.0 \%)$ & $72(13.6 \%)$ & 71 (19.5\%) & 0.018 \\
\hline Academic hospital & $732(81.7 \%)$ & 447 (84.2\%) & $285(78.1 \%)$ & 0.020 \\
\hline AKI stage at peak & & & & $<0.001$ \\
\hline 1 & $120(13.4 \%)$ & $48(9.0 \%)$ & $72(19.7 \%)$ & \\
\hline 2 & $130(14.5 \%)$ & $54(10.2 \%)$ & $76(20.8 \%)$ & \\
\hline 3 & $646(72.1 \%)$ & $429(80.8 \%)$ & $217(59.5 \%)$ & \\
\hline Admission dept. & & & & $<0.001$ \\
\hline ICU & $378(42.2 \%)$ & $258(48.6 \%)$ & $120(32.9 \%)$ & \\
\hline Renal & $160(17.9 \%)$ & $127(23.9 \%)$ & $33(9.0 \%)$ & \\
\hline Surgical & $104(11.6 \%)$ & $53(10.0 \%)$ & $51(14.0 \%)$ & \\
\hline Other & $254(28.3 \%)$ & $93(17.5 \%)$ & $161(44.1 \%)$ & \\
\hline Renalreferral & $518(57.8 \%)$ & $362(68.2 \%)$ & $156(42.7 \%)$ & $<0.001$ \\
\hline Cost, US dollars & $7190(3346,16431)$ & $9491(4284,18905)$ & $4537(2405,11036)$ & $<0.001$ \\
\hline Classification & & & & 0.048 \\
\hline Pre-renal & $345(38.5 \%)$ & $185(34.8 \%)$ & $160(43.8 \%)$ & \\
\hline Intra-renal & $437(48.8 \%)$ & $276(52.0 \%)$ & $161(44.1 \%)$ & \\
\hline Post-renal & 87 (9.7\%) & $52(9.8 \%)$ & $35(9.6 \%)$ & \\
\hline Unclassified & $27(3.0 \%)$ & $18(3.4 \%)$ & $9(2.5 \%)$ & \\
\hline Non-Oliguria & $368(48.7 \%)$ & $194(43.1 \%)$ & $174(57.0 \%)$ & $<0.001$ \\
\hline Severe comorbidity & $516(57.6 \%)$ & $269(50.7 \%)$ & $247(67.7 \%)$ & $<0.001$ \\
\hline $\begin{array}{l}\text { Renal recovery } \\
(n=615)\end{array}$ & & & & 0.094 \\
\hline Full recovery & $126(20.5 \%)$ & $71(17.9 \%)$ & $55(25.2 \%)$ & \\
\hline Partial recovery & $203(33.0 \%)$ & $134(33.8 \%)$ & 69 (31.7\%) & \\
\hline Failed recovery & $286(46.5 \%)$ & $192(48.4 \%)$ & $94(43.1 \%)$ & \\
\hline $\begin{array}{l}\text { Treatment withdrawal } \\
(n=859)\end{array}$ & $200(23.3 \%)$ & 99 (19.4\%) & $101(28.9 \%)$ & 0.001 \\
\hline In hospital Mortality & $216(25.1 \%)$ & $94(18.4 \%)$ & $122(35.0 \%)$ & $<0.001$ \\
\hline
\end{tabular}

Abbreviations: RRT renal replacement therapy, GPD gross domestic product, HA-AKI hospital acquired-acute kidney injury, CKD chronic kidney disease, $H T$ hypertension, $D M$ diabetes mellitus, CVD cardiovascular disease, ICU intensive care unit

${ }^{a}$ GDP per head was divided into tertiles calculated from the whole survey population

departments were $42.2 \%, 17.9 \%, 11.6 \%$ and $28.3 \%$ respectively. Most of the patients (72.1\%) had AKI stage 3, and the rest were at AKI stage $2(14.5 \%)$ or stage 1 (13.4\%), with $516(57.6 \%)$ patients having severe comorbidities. Only $57.8 \%$ of the patients $(n=518)$ had renal referral. There were 216 patients $(216 / 859,25.1 \%)$ died during their hospital stay (37 patients without identifiable outcome) and $200(200 / 859,23.3 \%)$ cases were 
recorded as 'treatment withdrawal'. Patients in male gender $(23.9 \%$ vs. $22.0 \%, p=0.515)$, with malignancies ( $28.7 \%$ vs. $22.3 \%, p=0.105$ ), with severe comorbidities (28.7\% vs. $16.2 \%, p<0.001)$, and from lower income areas $(24.5 \%$ tertile 1 vs. $27.1 \%$ tertile 2 vs. $17.5 \%$ tertile3, $p=0.020)$ were more likely to withdraw treatment. Among the survived patients who had identifiable record for renal recovery at discharge $(n=615), 126$ patients (20.5\%) got full renal recovery and 203 cases (33.0\%) got partial recovery.

\section{Comparison between participants with RRT or no-RRT treatment}

Of the 896 patients that had indications for RRT, only 531 cases $(59.3 \%)$ received RRT (Table 1). Compared with those who did not receive RRT, patients that were treated were younger $(57.0 \pm 18.2$ vs. $63.7 \pm 17.4$, $p<0.001)$, less male predominant $(60.6 \%$ vs. $72.6 \%$, $p<0.001$ ), and with less pre-existing cardiovascular disease $(26.0 \%$ vs. $35.3 \%, p=0.003)$. They were also featured as more of oliguria ( $56.9 \%$ vs. $43.0 \%, p<0.001)$, reaching higher AKI stage at peak $(80.8 \%$ vs. $59.5 \%$ at stage $3, p<0.001$ ), and having higher intra-renal AKI proportion $(52.0 \%$ vs. $44.1 \%, p=0.048)$, whereas with less patients having severe comorbidities $(50.7 \%$ vs. $67.7 \%, p<0.001)$. RRT was performed more in academic hospitals than in local hospitals $(61.1 \%$ vs. $51.2 \%$, $p=0.020)$, more in renal department $(79.4 \%)$ than in ICU $(68.3 \%)$, surgical $(51.0 \%)$ or other medical departments $(36.6 \%, p<0.001)$, and more in patients whose AKI being diagnosed by the doctors in charge or via nephrology consultation (69.9\% vs. $44.7 \%, p<0.001)$ than those who did not have. Patients from the lower income areas received RRT less frequently than those from the high-income areas $(p<0.001)$. Thein-hospital allcause mortality $(18.4 \%$ vs. $35.0 \%, p<0.001)$ and the proportion of "treatment withdrawal" $(19.4 \%$ vs. $28.9 \%$, $p=0.001$ ) was lower, and the in-hospital cost was higher in the patients who received RRT as compared to those who did not $(p<0.001)$. For the patients survived at discharge, renal recovery was not significantly different between those with and without RRT treatment $(p=0.094)$.

\section{Factors associated with no-RRT treatment in patients with RRT indications}

Multiple logistic regression analysis (Table 2) showed that factors that might be associated with no-RRT treatment were older age $(\mathrm{OR}=1.41$ per 10 years older, $95 \% \mathrm{CI}$ $1.18-1.70, p<0.001)$, male gender $(\mathrm{OR}=1.57,95 \% \mathrm{CI}$ $1.02-2.23, p=0.012)$, from lower income areas $(\mathrm{OR}=1.86$ tertile2, 95\% CI 1.21-2.86, $p=0.005$; OR $=3.08$ tertile1, 95\% CI 2.02-4.68, $p<0.001)$, hospital-acquired AKI $(\mathrm{OR}=1.28,95 \% \mathrm{CI} 0.98-1.82, p=0.172)$, non-oliguric AKI $(\mathrm{OR}=1.65,95 \%$ CI $1.19-2.30, p=0.003)$, and severe
Table 2 Multiple logistic regression of non-RRT treatment

\begin{tabular}{lll}
\hline Covariate & OR & $95 \% \mathrm{Cl}$ \\
\hline Age (per 10 years older) & $1.41(1.18-1.70)$ & $<0.001$ \\
Male (vs. female) & $1.57(1.10-2.23)$ & 0.012 \\
GDP per head & & \\
$\quad$ Tertile1 & $3.08(2.02-4.68)$ & $<0.001$ \\
Tertile2 & $1.86(1.21-2.86)$ & 0.005 \\
$\quad$ Tertile3 & Reference & \\
Academic hospital & $0.53(0.35-0.81)$ & 0.004 \\
Renal referral & $0.40(0.28-0.56)$ & $<0.001$ \\
AKI peak stage & & \\
$\quad$ Stage 1 & Reference & \\
Stage2 & $1.08(0.58-1.99)$ & 0.816 \\
$\quad$ Stage 3 & $0.53(0.32-0.88)$ & 0.013 \\
HA-AKl & $1.28(0.90-1.82)$ & 0.172 \\
Non-oliguria & $1.65(1.19-2.30)$ & 0.003 \\
Severe comorbidities & $1.60(1.12-2.28)$ & 0.010 \\
\hline
\end{tabular}

Abbreviations: RRT renal replacement therapy, GPD gross domestic product, $A K I$ acute kidney injury, HA-AKI hospital acquired-acute kidney injury

Multivariate logistic regression was adjusted for age (every 10 year increment), gender (female as reference), income (tertile 3 as reference), academic hospital (yes vs. no), renal referral (yes vs. no), AKI peak stage (Stage1 as reference),

HA-AKI (yes vs. no), Non-oliguria (yes vs. no), severe comorbidities (yes vs. no)

comorbidities $(\mathrm{OR}=1.60,95 \% \mathrm{CI} 1.12-2.28, p=0.010)$. Patients with AKI stage 3 at peak (OR $=0.53,95 \% \mathrm{CI}$ $0.32-0.88, p=0.013)$, admitted in academic hospitals $(\mathrm{OR}=0.53,95 \%$ CI $0.35-0.81, p=0.004)$ or had renal referral $(\mathrm{OR}=0.40,95 \%$ CI $0.28-0.56, p<0.001)$ tended to be at lower risk of no-RRT treatment.

Risk factors associated with in-hospital mortality (Table 3) Multiple logistic regression analysis showed that older age $(\mathrm{OR}=1.61$ per 10 years older, $95 \% \mathrm{CI} 1.26-2.05$, $p<0.001)$, severe comorbidities ( $\mathrm{OR}=3.85,95 \% \mathrm{CI}$ $2.38-6.23, p<0.001)$, hospital acquired AKI (OR = 3.00, 95\% CI 1.98-4.56, $p<0.001$ ), and higher AKI stage at peak (stage 3 vs. stage $1, \mathrm{OR}=2.20,95 \%$ CI 1.14-4.25, $p=0.019)$ were independent risk factors for in-hospital mortality, while RRT treatment $(\mathrm{OR}=0.58,95 \% \mathrm{CI}$ $0.38-0.89, p=0.013)$ and renal referral were protective factors against in-hospital death $(\mathrm{OR}=0.54,95 \% \mathrm{CI}$ $0.36-0.82, p=0.004$ ).

\section{RRT modalities}

The modalities of RRT included continuous renal replacement therapy (CRRT) in 286 cases (53.9\%), CRRT complemented by intermittent hemodialysis (IHD) in 33 cases (6.2\%), CRRT complemented by peritoneal dialysis (PD) in 4 cases (0.8\%), IHD only in 202 cases (38.0\%), and PD only in 6 cases (1.1\%) (Table 4). Nearly 3/4(233/ 323, 72.1\%) of CRRT (CRRT/CRRT + IHD/CRRT + PD) was performed in the ICU. The modalities of RRT varied 
Table 3 multiple logistic regression of in-hospital mortality

\begin{tabular}{lll}
\hline Covariate & OR(95\% Cl) & $P$ \\
\hline Age (per 10 years older) & $1.61(1.26-2.05)$ & $<0.001$ \\
Male & $1.39(0.90-2.14)$ & 0.139 \\
Income & & \\
$\quad$ Tertile1 & $0.80(0.48-1.33)$ & 0.390 \\
$\quad$ Tertile 2 & $0.66(0.40-1.09)$ & 0.105 \\
Academic hospital & $1.13(0.68-1.88)$ & 0.648 \\
Renal referral & $0.54(0.36-0.82)$ & 0.004 \\
AKI peak stage & & \\
$\quad$ Stage2 & $1.97(0.94-4.13)$ & 0.074 \\
$\quad$ Stage 3 & $2.20(1.14-4.25)$ & 0.019 \\
HA-AKI & $3.00(1.98-4.56)$ & $<0.001$ \\
CVD & $1.53(0.99-2.35)$ & 0.055 \\
DM & $1.48(0.92-2.37)$ & 0.104 \\
Non-oliguria & $0.68(0.45-1.02)$ & 0.059 \\
Severe comoridities & $3.85(2.38-6.23)$ & $<0.001$ \\
RRT & $0.58(0.38-0.89)$ & 0.013 \\
\hline
\end{tabular}

Multivariate logistic regression was adjusted for age (every 10 year increment), gender (female as reference), income (tertile 3 as reference), academic hospital (yes vs. no), renal referral (yes vs. no), AKI peak stage (Stage1 as reference),

HA-AKI (yes vs. no), CVD (yes vs. no), DM (yes vs. no),non-oliguria (yes vs. no), severe comorbidities (yes vs. no) and RRT (yes vs. no)

Abbreviations: $A K I$ acute kidney injury, $H A-A K I$ hospital acquired-acute kidney injury, CVD cardiovascular disease, $D M$ diabetes mellitus, $R R T$ renal replacement therapy

among different departments. There was a greater proportion of CRRT (CRRT/CRRT + IHD/CRRT + PD) in the ICU than in the renal or other departments $(90.3 \%$ vs. $22.0 \%$ vs. $42.5 \%, p<0.001)$, and a greater proportion of IHD in the renal department than that in the ICU or other departments $(76.4 \%$ vs. $9.3 \%$ vs. $55.5 \%, p<0.001)$. PD was not compared among different departments because of the low frequency of less than 5 .

\section{RRT modalities in patients with or without CRRT indications}

Among the patients who were prescribed CRRT $(n=323)$, 204 cases $(63.2 \%)$ had CRRT indications that included at least one of the comorbidities such as rhabdomyolysis, sepsis, multiple organ dysfunction syndrome (MODS), respiratory failure needing mechanical ventilation, or shock. On the other hand, of the 250patients who had CRRT indications, 46 cases $(18.4 \%)$ were treated with IHD, among whom 9 cases (9/46, 20\%) left hospital with "treatment withdrawal" soon afterwards, indicating an influence of economic conditions on the choice of RRT treatment.

Among the 119 patients that had no CRRT indications but prescribed with CRRT, half ( $n=60,50.4 \%)$ were hospitalized in ICU and the others were admitted to renal ( $n=22,18.5 \%)$, surgical $(n=6,5.0 \%)$ and other medical units $(n=31,26.1 \%)$. Among the various clinical units, more patients that had no CRRT indications were treated with CRRT in the ICU $(60 / 71,84.5 \%)$ compared with those in renal $(22 / 110,20.0 \%)$, surgical $(6 / 33,18.2 \%)$ and other medical units $(31 / 67,46.3 \%),(p<0.001)$.

Of the 281 patients that had no CRRT indications, renal referrals were lower in the CRRT prescriptions than the IHD prescriptions $(67.2 \%$ vs. $84.6 \%, p=0.001)$. There was no significant difference in the AKI peak stage or the proportion of severe comorbidities between the patients with CRRT treatment $(n=119)$ and those with other RRT treatments $(n=162)$, whereas the inhospital mortality rate $(10.6 \%$ vs. $4.4 \%, p=0.047)$ and the overall medical costs $(7944[4248,16,055]$ vs. $5100[2948,9396]$ US dollars, $p<0.001)$ were higher in the patients with CRRT treatment (Table 5).

\section{Discussion}

There is an increasing incidence and prevalence of AKI globally $[1,7,11]$ and severe AKI is associated with increased mortality up to greater than 50\% [12-14]. In the absence of effective pharmacologic interventions for severe AKI, renal replacement therapy remains the main supportive management, and therefore is one of the critical aspects for improvement to achieve the goal of "ISN AKF 0 by 25 ". However, up to now little is known about the need, availability, and maneuverability of RRT in the clinical practice in developing countries. Based on the

Table 4 RRT-modalities

\begin{tabular}{lllll}
\hline Clinical units & All $(n=531)$ & ICU $(n=258)$ & Renal $(n=127)$ & Others $(n=146)$ \\
\hline $\begin{array}{llll}\text { RRT modalities } \\
\text { CRRT }\end{array}$ & $286(53.9 \%)$ & $221(85.7 \%)$ & $18(14.2 \%)$ & $47(32.2 \%)$ \\
CRRT + IHD & $33(6.2 \%)$ & $11(4.3 \%)$ & $8(6.3 \%)$ & $14(9.6 \%)$ \\
CRRT + PD & $4(0.8 \%)$ & $1(0.4 \%)$ & $2(1.6 \%)$ & $1(0.7 \%)$ \\
IHD & $202(38.0 \%)$ & $24(9.3 \%)$ & $97(76.4 \%)$ & $81(55.5 \%)$ \\
PD & $6(1.1 \%)$ & $1(0.4 \%)$ & $2(1.6 \%)$ & $3(2.1 \%)$ \\
\hline
\end{tabular}

Abbreviations: $R R T$ renal replacement therapy, $C R R T$ continuous renal replacement therapy, IHD intermittent hemodialysis, $P D$ peritoneal dialysis, ICU intensive care unit

${ }^{*} \mathrm{CRRT}+\mathrm{PD}$ and PD were not included in the Chi-square analysis because of the low frequency less than 5 
Table 5 New Comparison between CRRT-treated and other-RRT-treated patients without CRRT indications

\begin{tabular}{|c|c|c|c|}
\hline & CRRT-treated $(n=119)$ & Other-RRT-treated $(n=162)$ & $P$ \\
\hline Age, years & $57.3 \pm 18.6$ & $54.6 \pm 16.6$ & 0.199 \\
\hline Male sex & $68(57.1 \%)$ & $87(53.7 \%)$ & 0.567 \\
\hline AKI stage at peak & & & 0.835 \\
\hline Stage 1 & $12(10.1 \%)$ & $13(8.0 \%)$ & \\
\hline Stage 2 & $8(6.7 \%)$ & $11(6.8 \%)$ & \\
\hline Stage 3 & 99 (83.2\%) & $138(85.2 \%)$ & \\
\hline Severe comorbidities & $11(9.2 \%)$ & $12(7.4 \%)$ & 0.579 \\
\hline Cost, US Dollars ${ }^{\mathrm{a}}$ & $7944(4248,16055)$ & $5100(2948,9396)$ & $<0.001$ \\
\hline In hospital mortality ${ }^{b}$ & $12(10.6 \%)$ & $7(4.4 \%)$ & 0.047 \\
\hline Treatment withdrawal $^{c}$ & $23(20.4 \%)$ & $8(5.0 \%)$ & $<0.001$ \\
\hline Renal-referral & $80(67.2 \%)$ & $137(84.6 \%)$ & 0.001 \\
\hline Admission dept. & & & $<0.001$ \\
\hline $\mathrm{ICU}$ & $60(50.4 \%)$ & $11(6.8 \%)$ & \\
\hline Renal & $22(18.5 \%)$ & $88(54.3 \%)$ & \\
\hline Surgical & $6(5.0 \%)$ & $27(16.7 \%)$ & \\
\hline Other & $31(26.1 \%)$ & $36(22.2 \%)$ & \\
\hline
\end{tabular}

Abbreviations: CRRT continuous renal replacement therapy, RRT renal replacement therapy, AKI acute kidney disease, ICU intensive care unit ${ }^{\mathrm{a}}$ Missing value 36, ${ }^{\mathrm{b}}$ Missing value $9,{ }^{\mathrm{C}}$ Missing value 9

nationwide survey of AKI in over 2 million adult hospitalizations, we were able to estimate the burden of RRT need and the real state of RRT performance in Mainland China [9].

Although there are some disagreements about the optimal timing for initiating RRT in AKI patients, there is no doubt that RRT should be performed in patients with life-threatening conditions including overt fluid imbalance, electrolyte abnormalities, acid-base disturbances, over accumulating metabolic toxins and uremic complications $[15,16]$. According to these traditional RRT indications, we found that $11.8 \%$ of the AKI patients were in needs of RRT. From what we have reported in this nationwide survey as about 2.9 million AKI cases hospitalized in Mainland China during 2013 [9], it can be estimated that at least 342,200 AKI patients needed RRT treatment during their hospitalization in 2013. However, around $40 \%$ of these AKI patients needing RRT did not receive the treatment, and had significantly increased risk for mortality compared with those who were treated with RRT. Why had these patients been undertreated? As all the hospitals that were enrolled in the nationwide survey have facilities for IHD and PD, the significant situation of RRT under treatment cannot be attributed to the shortage of medical resources. We then tried to disclose potential affecting factors associated with patient and medical staff.

From multi-factorial analysis, we found that if the patients were older, in male gender, located in lower income areas, from local hospitals, or with malignancies and other severe comorbidities, they were more likely to be RRT under treated. This implies that socio-economic status might strongly affect the treatment choice for the individuals, and more consideration from the family would be made when their older and more severe relatives are in needs of RRT treatment. As male patients usually are the main support of their families, their sickness would directly affect the economic status of the family, and therefore the high medical cost for RRT treatment might be unaffordable. Besides, in some rural areas and minority regions, patients would prefer dying at home rather than in hospital, which could also contribute to the lack of RRT treatment in local hospitals. The high "treatment withdrawal" rate in these patients reinforces the above findings. Medical staff involved in patient care also played important roles in the current clinical status of RRT treatment selection. Patients who had oliguric AKI or stage 3 AKI, where the situation of renal failure easily arouses doctors' attention, were of less risk for RRT under treatment. Moreover, those who were treated in renal department or received renal consultation had been significantly protected from RRT under treatment, which emphasizes the important impact of nephrologists on the treatment strategy determination in severe AKI patients.

Despite the significant under-treatment of RRT indicated patients in this survey, there was potential inappropriate prescription of CRRT treatment, which is an advanced but costy technique in treating critically ill patients. Since studies have shown that CRRT could benefit 
patients with sepsis, unstable hemodynamic, acute respiratory distress syndrome needing mechanical ventilation, MODS, and rhabdomyolysis [17-25], we then expanded the indications for CRRT according to these clinical conditions. We found that more than $1 / 3$ of the patients were prescribed CRRT with unrecognized indication, of whom the survival was not improved while the cost was higher as compared to those treated with other RRT modalities. Therefore, CRRT could have been over prescribed in the clinical practice. What might be the reasons? Firstly, in the majority of the studied hospitals, CRRT facility is the only available RRT modality in the ICU departments and is performed by the ICU teams, therefore it is much more convenient to initiate CRRT than calling the nephrology team or transferring the patients to hemodialysis units. This can partially explain why most CRRT was prescribed in ICU departments. The fact that renal referral was associated with lower percentage of CRRT prescription in those patients who had no CRRT indications supports this possibility. Secondly, there could be a large variability in the understanding and decision of RRT modalities among different clinicians. The indications for CRRT have not been well established, and the implementation is more dependent on personal experience and preference in some circumstances. Besides, the very limited use of PD (only $1 \%$ in the current survey although with $100 \%$ availability in the study hospitals) makes CRRT the first choice in patients with unstable hemodynamic conditions. Investigations about the choice of RRT modalities in clinical practitioners would be helpful to disclose the real factors that influence their decisions, and enables improvements in the performance.

Therefore, the current study disclosed a noticeable under treatment rate in RRT indicated AKI patients, meanwhile for those who had been prescribed RRT, over treatment with CRRT could have also existed, which raises an important issue of rational use of medical resources. Although all of the hospitals in this study are capable of RRT, there still had been patients that could not afford RRT treatment, not to mention the numerous hospitals in rural areas that have no RRT resources. This is a common phenomenon in developing countries [26-28]. Therefore financial support from the government is in great need for helping the unaffordable conditions. On the other hand, proper utilization of RRT modalities, such as rational prescription of CRRT and more performance of $\mathrm{PD}$, would help to increase the medical economics and save more lives of severe AKI with the limited medical resources. To achieve this goal, education and training for RRT practitioners is of most importance.

There are some limitations in this study. Firstly, as a retrospective study, we could not clearly clarify the causal relationship between treatments and outcomes.
Secondly, classifications of clinical situation were made only on the basis of the medical records, thus the real clinical practice could not be fully recalled and biases may occur, such as underestimating of CRRT indicated patients. Finally, the inadequate measurement of $\mathrm{SCr}$ may underestimate the true need for RRT and renal recovery may not be able to compare with high missing value. However, this nation-wide survey is the largest and most representative of survey in AKI patients in China, from which we are able to reveal the current RRT situation in China and present some potential similarities in other developing countries.

\section{Conclusions}

In summary, RRT has improved mortality in patients with severe AKI, however there have been a significant under treatment of RRT in China based on current retrospective study. CRRT might have been overprescribed and the modalities of RRT need to be optimized to improve the medical economic efficiency in China. The situation that has been revealed in this study may represent a common status of RRT choice and performance in the real world of clinical practice in developing countries, calling for the awareness of appropriate utilization of RRT in severe AKI, a global, burdensome but treatable disease.

\begin{abstract}
Abbreviations
AKl: Acute kidney injury; CKD: Chronic kidney disease; CRRT: Continuous renal replacement therapy; CVD: Cardiovascular disease; DM: Diabetes mellitus; GDP: Gross domestic product; ICU: Intensive care unit; IHD: Intermittent hemodialysis; ISN: International Society of Nephrology; MODS: Multiple organ dysfunction syndrome; PD: Peritoneal dialysis; RRT: Renal replacement therapy; SCr: Serum creatinine
\end{abstract}

\section{Acknowledgements}

We thank the members of the ISN AKF 0 by 25 China Consortium (Prof Li Yang, Prof G Xing, Prof L Wang, Prof Y Wu, Prof Suhua Li, Prof G Xu, Prof Q He, Prof J Chen, Prof M Chen, X Liu, Prof Z Zhu, Prof Lin Yang, Prof X Lian, Prof F Ding, Prof $Y$ Li, Prof Huamin Wang, Prof Jianqin Wang, Prof R Wang, Prof C Mei, Prof Jixian Xu, Prof R Li, Prof J Cao, L Zhang, Yan Wang, Jinhua Xu, Prof B Bao, Prof Bicheng Liu, Prof H Chen, Prof Shaomei Li, Prof Y Zha, Prof Q Luo, D Chen, Prof Y Shen, Prof Y Liao, Z Zhang, X Wang, K Zhang, L Liu, Prof P Mao, C Guo, J Li, Prof Z Wang, S Bai and S Shi).

We thank our respectable professor Haiyan Wang for her guidance of this work. We thank Professor VladoPerkovic, Professor Martin Gallagher, Professor Meg Jardine, Dr. Amanda Ying Wang and Dr. Vivek Kumar for their review and comments.

\section{Funding}

This study was supported by National Project 985 to Peking University for Clinical Study on Cooperation; Beijing Training Program for Talents (20110009001000002); National Natural Science Foundation of China (81270777); National Natural Science Foundation of China (81500575), International Society of Nephrology Research Committee; Bethune Fund Management Committee. The study was designed, conducted, analyzed and interpreted by the investigators independent of sponsors.

Availability of data and materials

The datasets during and/or analysed during the current study are available from the corresponding author on reasonable request. 


\section{Authors' contribution}

Conceived and designed the experiments: FW DH YW LW LY. Performed the experiments: FW DH YW. Analyzed the data: YW DH. Contributed to the writing of the manuscript: FW DH YW YF LW LY. All authors have read, and confirm that they meet ICMJE criteria for authorship. Wrote the first draft of the manuscript: DH FW LY. Agree with the manuscript's results and conclusions: FW DH YW YF LW LY. ISN AKF 0 by 25 China Consortium members collected the data. All authors read and approved the final manuscript.

\section{Competing interests}

The authors declare that they have no competing interests.

\section{Consent for publication}

The manuscript contains no individual person's data in any form.

\section{Ethics approval and consent to participate}

The study was approved by the ethic committees of Peking University First Hospital and the enrolled study hospitals. Due to the retrospective nature of the study, written informed consent for participation was waived.

\section{Publisher's Note}

Springer Nature remains neutral with regard to jurisdictional claims in published maps and institutional affiliations.

\section{Author details}

'Division of Nephrology, Sichuan Academy of Medical Sciences \& Sichuan Provincial People's Hospital, Chengdu 610072, China. ${ }^{2}$ Renal Division, Department of Medicine, Peking University First Hospital, Beijing 100034, People's Republic of China. ${ }^{3}$ Key Laboratory of Renal Disease, Ministry of Health of China, Beijing 100034, People's Republic of China.

Received: 15 January 2017 Accepted: 22 April 2017

Published online: 04 May 2017

\section{References}

1. Lewington AJ, Cerda J, Mehta RL. Raising awareness of acute kidney injury: a global perspective of a silent killer. Kidney Int. 2013;84(3):457-67.

2. Lafrance JP, Miller DR. Acute kidney injury associates with increased longterm mortality. J Am Soc Nephrol. 2010;21(2):345-52.

3. Chertow GM, Burdick E, Honour M, Bonventre JV, Bates DW. Acute kidney injury, mortality, length of stay, and costs in hospitalized patients. J Am Soc Nephrol. 2005;16(11):3365-70.

4. Fang $Y$, Ding $X$, Zhong Y, Zou J, Teng J, Tang Y, Lin J, Lin P. Acute kidney injury in a Chinese hospitalized population. Blood Purif. 2010;30(2):120-6.

5. Ali T, Khan I, Simpson W, Prescott G, Townend J, Smith W, Macleod A. Incidence and outcomes in acute kidney injury: a comprehensive populationbased study. J Am Soc Nephrol. 2007;18(4):1292-8.

6. Hsu CY, McCulloch CE, Fan D, Ordonez JD, Chertow GM, Go AS. Communitybased incidence of acute renal failure. Kidney Int. 2007;72(2):208-12.

7. Susantitaphong P, Cruz DN, Cerda J, Abulfaraj M, Alqahtani F, Koulouridis I, Jaber BL, Acute Kidney Injury Advisory Group of the American Society of N. World incidence of AKI: a meta-analysis. Clin J Am Soc Nephrol. 2013;8(9): 1482-93.

8. Cerda J, Bagga A, Kher V, Chakravarthi RM. The contrasting characteristics of acute kidney injury in developed and developing countries. Nat Clin Pract Neprhol. 2008;4(3):138-53.

9. Yang L, Xing G, Wang L, Wu Y, Li S, Xu G, He Q, Chen J, Chen M, Liu X, et al. Acute kidney injury in China: a cross-sectional survey. Lancet. 2015;386(10002): 1465-71.

10. Kidney Disease: Improving Global Outcomes (KDIGO) Acute Kidney Injury Work Group. KDIGO clinical practice guideline for acute kidney injury. Kidney Int Suppl. 2012;2:1-138.

11. Kam Tao Li P, Burdmann EA, Mehta RL, World Kidney Day Steering C. Acute kidney injury: global health alert. Kidney Int. 2013;83:372-6.

12. Uchino $\mathrm{S}$. The epidemiology of acute renal failure in the world. Curr Opin Crit Care. 2006;12(6):538-43.

13. Clermont G, Acker CG, Angus DC, Sirio CA, Pinsky MR, Johnson JP. Renal failure in the ICU: comparison of the impact of acute renal failure and endstage renal disease on ICU outcomes. Kidney Int. 2002;62(3):986-96.
14. Liangos O, Wald R, O'Bell JW, Price L, Pereira BJ, Jaber BL. Epidemiology and outcomes of acute renal failure in hospitalized patients: a national survey. Clin J Am Soc Nephrol. 2006;1(1):43-51.

15. Ricci Z, Ronco C. Timing, dose and mode of dialysis in acute kidney injury. Curr Opin Crit Care. 2011;17(6):556-61.

16. Palevsky PM. Renal replacement therapy in acute kidney injury. Adv Chronic Kidney Dis. 2013;20(1):76-84.

17. Rabindranath K, Adams J, Macleod AM, Muirhead N. Intermittent versus continuous renal replacement therapy for acute renal failure in adults. Cochrane Database Syst Rev. 2007;3:CD003773.

18. Fieghen $\mathrm{HE}$, Friedrich JO, Burns KE, Nisenbaum R, Adhikari NK, Hladunewich MA, Lapinsky SE, Richardson RM, Wald R, University of Toronto Acute Kidney Injury Research $\mathrm{G}$. The hemodynamic tolerability and feasibility of sustained low efficiency dialysis in the management of critically ill patients with acute kidney injury. BMC Nephrol. 2010;11:32.

19. Zeng X, Zhang L, Wu T, Fu P. Continuous renal replacement therapy (CRRT) for rhabdomyolysis. Cochrane Database Syst Rev. 2014;6:CD008566.

20. Yao LQ, Jin ZC, Ji MS, Xia CY, Yu ZX, Liu J, Hu XL, Yan J. Effect of continuous renal replacement therapy started at different time on patients with multiple organ dysfunction syndrome. Zhonghua Yi Xue Za Zhi. 2011;91(24): 1663-7.

21. Joannidis M. Continuous renal replacement therapy in sepsis and multisystem organ failure. Semin Dial. 2009;22(2):160-4.

22. Servillo G, Vargas M, Pastore A, Procino A, lannuzzi M, Capuano A, Memoli A, Riccio E, Memoli B. Immunomodulatory effect of continuous venovenous hemofiltration during sepsis: preliminary data. Biomed Res Int. 2013:2013:108951.

23. Davenport A. Continuous renal replacement therapies in patients with acute neurological injury. Semin Dial. 2009;22(2):165-8.

24. Ronco C, Bellomo R, Brendolan A, Pinna V, La Greca G. Brain density changes during renal replacement in critically ill patients with acute renal failure. Continuous hemofiltration versus intermittent hemodialysis. J Nephrol. 1999; 12(3):173-8.

25. Han F, Sun R, Ni Y, Hu X, Chen X, Jiang L, Wu A, Ma L, Chen M, Xv Y, et al. Early initiation of continuous renal replacement therapy improves clinical outcomes in patients with acute respiratory distress syndrome. Am J Kidney Dis. 2015;349(3):199-205

26. Barsoum RS. Chronic kidney disease in the developing world. N Engl J Med. 2006;354(10):997-9.

27. Dirks JH, Levin NW. Dialysis rationing in South Africa: a global message. Kidney Int. 2006;70(6):982-4

28. Moosa MR, Kidd M. The dangers of rationing dialysis treatment: the dilemma facing a developing country. Kidney Int. 2006;70(6):1107-14.

\section{Submit your next manuscript to BioMed Central and we will help you at every step:}

- We accept pre-submission inquiries

- Our selector tool helps you to find the most relevant journal

- We provide round the clock customer support

- Convenient online submission

- Thorough peer review

- Inclusion in PubMed and all major indexing services

- Maximum visibility for your research

Submit your manuscript at www.biomedcentral.com/submit
) Biomed Central 